\title{
EXTENSIONS OF ORTHOMORPHISMS
}

\author{
A. W. WICKSTEAD \\ (Received 17 November 1978; revised 27 August 1979) \\ Communicated by E. Strzelecki
}

\begin{abstract}
We consider, on an Archimedean Riesz space, the spaces of all linear operators lying between two multiples of the identity (for the order), those leaving all ideals invariant and the order bounded orthomorphisms. We find, if $E$ is uniformly complete, necessary and sufficient conditions for all such operators defined on sublattices of $E$ to extend to the whole of $E$. Examples are given to show the role of uniform completeness. For the space of all orthomorphisms we give a sufficient condition on $E$ for such an extension to exist.
\end{abstract}

1980 Mathematics subject classification (Amer. Math. Soc.): 47 B 55.

\section{Introduction}

Let $E$ be an Archimedean Riesz space (we follow Luxemburg and Zaanen (1971) for general Riesz space terminology). We let $\operatorname{Stab}(E)$ denote the space of all orthomorphisms on $E$ (see below for all definitions) and $P(E)$ denote the subspace of order bounded orthomorphisms. We also use $S(E)$ to denote the space of all linear operators on $E$ which leave all ideals invariant and $Z(E)$ to denote those linear operators $T$ on $E$ for which there is $\lambda \geqslant 0$ with $-\lambda x \leqslant T x \leqslant \lambda x$ for all $x \in E^{+}$.

It is known that $P(E) \neq \operatorname{Stab}(E)$ in general. At one time the author conjectured that $P(E)=\operatorname{Stab}(E)$ when $E$ is Dedekind complete. In an attempt to disprove this the author needed to extend an orthomorphism from an Archimedean Riesz space to its universal completion (Luxemburg and Zaanen (1971), Definition 50.4). This led him to ask when such extensions are possible, for each of the four spaces mentioned above.

Given an Archimedean Riesz space $E$ and a sublattice $H$ of $E$ it is not easy to describe even when each element of $Z(H)$ extends to an element of $Z(E)$ (the simple case $E=C(X)$ will give some idea of the difficulties involved). A more 
tractable problem is to seek those $E$ for which the appropriate operators extend, for any sublattice $H$. For the spaces $Z(\cdot), S(\cdot)$ and $P(\cdot)$ we have a necessary and sufficient condition, at least when $E$ is uniformly complete (when this assumption is used, examples are given to show that it cannot be dispensed with). For Stab(·), however, we have only a sufficient condition. It seems to the author that there is little hope of deciding whether or not this condition is necessary until rather more is known about the structure of orthomorphisms which are not order-bounded.

Section 2 contains the definitions of the spaces involved together with some relevant results of representation theory. The extension results are all in Section 3.

\section{Orthomorphisms and representations}

Let $E$ be an Archimedean Riesz space. A linear operator $T$ on $E$ is called an orthomorphism if $T x \perp y$ whenever $x \perp y$. An orthomorphism is order bounded (in the sense that it maps order bounded sets to order bounded sets) if and only if it is the difference of two positive orthomorphisms (see Meyer (1977), Corollaire 2.4). We follow Meyer by writing $\operatorname{Stab}(E)$ for the space of all orthomorphisms on $E$, but retain the notation of Wickstead (1977) in writing $P(E)$ for the space of all order bounded orthomorphisms on $E$. We also retain the use of $S(E)$ to denote the space of all linear operators on $E$ which leave every ideal invariant and $Z(E)$ to denote those linear operators $T$ on $E$ for which there exists $\lambda \geqslant 0$ with $-\lambda x \leqslant T x \leqslant \lambda x$ for each $x \in E^{+}$.

There are always inclusions

$$
Z(E) \subset S(E) \subset P(E) \subset S t a b(E)
$$

and in general all three inclusions may be proper (see Wickstead (1977) for the first two and Meyer (1977), Example 2.6 for the third. A similar example has been communicated to the author by Luxemburg (1977). That the inclusion may be proper will also follow from example (f) below).

If $S$ is a topological space then $C^{\infty}(S)$ denotes the continuous extended real valued functions on $S$ which are finite except on a nowhere dense set. In general this will not be a linear space when we define $h=f+g$ to mean that $h(s)=f(s)+g(s)$ whenever the sum is defined (we shall say that sums of the form $\infty+(-\infty)$ and products of the form $0 \cdot( \pm \infty)$ are not defined). If $S$ is extremally disconnected then $C^{\infty}(S)$ is a linear space and even an algebra when we define $f g(s)=f(s) g(s)$ whenever the product is defined (see Luxemburg and Zaanen (1971), Theorem 47.2).

We say that the map $x \mapsto x^{\wedge}$ of $E$ into $C^{\infty}(S)$ is a representation of $E$ if

(i) $E^{\wedge}=\left\{x^{\wedge}: x \in E\right\}$ is a linear space and sublattice of $C^{\infty}(S)$.

(ii) $x \mapsto x^{\wedge}$ is a Riesz space isomorphism of $E$ onto $E^{\wedge}$.

If, further, 
(iii) For each $s \in S$ there is $x \in E$ with $0<x^{\wedge}(s)<\infty$, we say that the representation is admissible.

Order bounded orthomorphisms and representations tie in together nicely. If $T \in P(E)$ and $x \rightarrow x^{\wedge}$ is a representation of $E$ in $C^{\infty}(S)$ then there is $\varphi \in C^{\infty}(S)$ such that, for each $x \in E$,

$$
(T x)^{\wedge}(s)=\varphi(s) x^{\wedge}(s)
$$

for those $s \in S$ for which the product is defined (see Wickstead (1977), Theorem 2.5 for a general proof and Bigard and Keimel (1969) or Conrad and Diem (1971) for a special case). Conversely any operator which can be described in this way lies in $P(E)$; so in particular the representation cannot be extended to $\operatorname{Stab}(E)$. Later this will account for the difference between our results for $\operatorname{Stab}(E)$ and the other spaces. It is, perhaps, worth recording here that, for $T \in P(E)$ and the corresponding $\varphi \in C^{\infty}(S), T \in S(E)$ if and only if $\varphi$ is bounded on the support of each $x^{\wedge}$ whilst $T \in Z(E)$ if and only if $\varphi$ is bounded.

Every Archimedean Riesz space has many admissible representations. It is not always possible, even assuming uniform completeness, to choose one so that $E^{\wedge}$ is an ideal in $C^{\infty}(S)$. This is possible (and we shall need this later) if $E$ is Dedekind complete, that is every nonempty subset with an upper bound has a least upper bound. Indeed let $\left(e_{\gamma}\right)_{\gamma \in \Gamma}$ be a maximal disjoint family of positive elements of $E$. We may use the Yosida representation (Luxemburg and Zaanen (1971), Section 42) to represent the ideal generated by $e_{\gamma}$ as $C\left(X_{\gamma}\right)$, where $X_{\gamma}$ is compact Hausdorff. As this ideal is Dedekind complete it follows from Luxemburg and Zaanen (1971), Theorem 43.11 that $X_{\gamma}$ is extremally disconnected. This representation may be extended to take the band generated by $e_{\gamma}$ into $C^{\infty}\left(S_{\gamma}\right)$. The natural representation of $E$ on the disjoint union of the $X_{\gamma}$ 's will have the desired property.

\section{Extension theorems}

Our first result not only deals with the problem of extensions for $Z(\cdot)$ but also will be referred to in the proof of Theorem 3 .

Recall that an Archimedean Riesz space has the projection property if for every band $B, B \oplus B^{d}$ is the whole of $E$. For each $e \in E_{+}$the expression

$$
\|x\|_{e}=\inf \{\lambda>0:-\lambda e \leqslant x \leqslant \lambda e\}
$$

defines a norm on the ideal generated by $e$. If each such ideal is complete for $\|\cdot\|_{e}$ then $E$ is uniformly complete.

THEOREM 1. Consider the following four conditions on an Archimedean Riesz space $E$. 
(i) E is Dedekind complete.

(ii) If $H$ is a sublattice of $E$ and $T \in Z(H)$ then there is $T \in Z(E)$ with $T \mid H=T$.

(iii) If $H$ is a sublattice of $E$ and $T \in Z(H)$ then there is $\bar{T} \in P(E)$ with $T \mid H=T$.

(iv) $E$ has the projection property.

In general (i) $\Rightarrow$ (ii) $\Rightarrow$ (iii) $\Rightarrow$ (iv). If $E$ is uniformly complete then all four conditions are equivalent.

ProOF. (i) $\Rightarrow$ (ii). It suffices to prove that each $T \in Z(H)^{+}$extends as required. Suppose also that $\lambda>0$ with $T h \leqslant \lambda h$ for all $h \in H^{+}$. Define $\varphi: E \rightarrow E$ by $\varphi(x)=\lambda x^{+}$, so that $\varphi$ is positive homogeneous and sublinear. As $T \leqslant \varphi$ on $H$ we may use the Hahn-Banach theorem to extend $T$ to $\bar{T}: E \rightarrow E$ with $0 \leqslant \bar{T} \leqslant \varphi$. Thus if $x \in E^{+}$, $0 \leqslant \bar{T} x \leqslant \lambda x$ and $\bar{T}$ is the required extension of $T$ in $Z(E)$.

(iii) $\Rightarrow$ (iv). Let $B$ be a band in $E$. Define $T$ on $B \oplus B^{d}$ to be linear and with $T \mid B$ being the identity on $B$ and $T \mid B^{d}$ to be the zero operator. Let $T \in Z(E)$ be an extension of $T$. This is order continuous and hence positive. Thus, if $x \in E^{+}$,

$$
\begin{aligned}
\bar{T} x & =\sup \left\{T g: 0 \leqslant g \leqslant f, g \in B \oplus B^{d}\right\} \\
& =\sup \{g: 0 \leqslant g \leqslant f, g \in B\}
\end{aligned}
$$

which is the definition of the band projection onto $B$.

Theorem 42.6 of Luxemburg and Zaanen (1971) asserts that Dedekind completeness is equivalent to the projection property together with uniform completeness. This suffices to complete the proof, as clearly (ii) $\Leftrightarrow$ (iii).

We give now two examples to show that the implications (ii) $\Rightarrow$ (i) and (iv) $\Rightarrow$ (ii) in Theorem 1 are not valid in the absence of the assumption of uniform completeness. We refer to (ii) as the $Z(\cdot)$-extension property. We first present a general construction.

Let $X$ be a completely regular Hausdorff space and $A$ be any linear space of continuous real valued functions on $X$. We say a function is locally in $A$ if

(i) The domain of $f, D(f)$, is a dense open subset of $X$.

(ii) If $x \in D(f)$ there is a neighbourhood $U$ of $x$ and $g \in A$ with $g|U=f| U$.

We define an equivalence relation on the set of all functions that are locally in $A$ by defining $f \equiv g$ to mean $f|D(f) \cap D(g)=g| D(f) \cap D(g)$. Let $L_{A}(X)$ denote the space of equivalence classes, under $\equiv$, of the functions which are locally in $A$.

$L_{A}(X)$ is a linear space if we define

$$
\begin{aligned}
D(\lambda f) & =D(f), \quad(\lambda f)(x)=\lambda \cdot f(x) \quad(x \in D(f)), \\
D(f+g) & =D(f) \cap D(g), \quad(f+g)(x)=f(x)+g(x) \quad(x \in D(f+g)),
\end{aligned}
$$

where, as usual, we do not bother to distinguish between functions and their 
equivalence classes. For the pointwise ordering $L_{A}(X)$ is a vector lattice which is Archimedean provided $X$ has the Baire property. For example, if we define $f \vee g$ by

$$
\begin{aligned}
& D(f \vee g)=[D(f) \cap D(g)] \backslash \partial\{x \in X: f(x)=g(x)\}, \\
& f \vee g(x)=f(x) \vee g(x) \quad(x \in D(f \vee g))
\end{aligned}
$$

then it is not difficult to check that $f \vee g$ is the supremum of $f$ and $g$ (divide $D(f \vee g)$ into three disjoint open sets on which $f<g, f=g$ and $f>g$ respectively to show that condition (ii) is satisfied).

A band $B$ in $L_{A}(X)$ is any set of the form $\left\{f \in L_{A}(X): f \mid Y \cap D(f) \equiv 0\right\}$ where $Y$ is a regular closed subset of $X$ (that is $Y$ is the closure of its own interior). Any space $L_{A}(S)$ has the projection property, for if $g \in L_{A}(X)$, let

$$
g_{1}\left|D(g)^{\circ} \cap Y \equiv 0, \quad g_{1}\right| D(g) \backslash Y \equiv g|D(g)| Y .
$$

Then $g_{1} \in B$ and $g-g_{1} \perp B$.

Any element $T \in P\left(L_{A}(X)\right)$ may be described by specifying that, for $f \in L_{A}(X)$,

$$
T f(x)=\varphi(x) f(x) \quad(x \in D(f) \cap D(\varphi))
$$

for some $\varphi \in L_{C(X)}(X)$. The proof of Theorem 2.5 of Wickstead (1977) may be repeated to show this, where the role of points where a function is infinite is now taken by points where the function is not defined.

$L_{A}^{b}(X)=\left\{f \in L_{A}(X): f\right.$ is bounded $\}$ is an ideal in $L_{A}(X)$ and thus also has the projection property. The representation result for order bounded orthomorphisms also works for this subspace.

(a) Archimedean Riesz space with the projection property but without the $Z(\cdot)$ extension property

Take $I=[0,1], G$ to be the space of all polynomial functions on $I, F$ the linear span of $G$ and the exponential function, exp, and $C$ the space of constant functions. Let $E=L_{F}^{b}(I)$ and $H$ be the sublattice $L_{G}^{b}(I)$. It is easy to check that we may identify $Z(H)$ with $H$ itself, in the sense that if $T \in Z(H)$ there is $h \in H$ with, for $f \in H$,

$$
T f(x)=h(x) f(x) \quad(x \in D(f) \cap D(h))
$$

(or see Theorem 3 of Zaanen (1975)). Now suppose $T$ extends to $\bar{T} \in Z(E)$. Clearly we still have

$$
T f(x)=h(x) f(x) \quad(x \in D(f) \cap D(h)),
$$

where now $f$ is any element of $E$. Consider $T(\exp )$. At each point $x \in D(h) \cap D(T(\exp ))$ there is a neighbourhood $U$ on which $T(\exp )$ coincides with $p+\alpha \cdot \exp$, where $p$ is a polynomial and $\alpha \in \mathbf{R}$. It follows that, on $U \cap D(h), h$ coincides with $(p / \exp )+\alpha$. There is a neighbourhood $V$ of $x$ on which $h$ coincides with $q$, a polynomial function. Thus on $U \cap V \cap D(h)$ the functions $q$ and $(p / \exp )+\alpha$ coincide. Thus, on 
$U \cap V \cap D(h), p \equiv 0$ and $q \equiv \alpha$. That is if $T$ is to extend to $T \in Z(E)$ then $h \in L_{C}^{b}(I)$. Clearly $L_{F}^{b}(I) \neq L_{C}^{b}(I)$ and so the $Z(\cdot)$-extension property fails for $E$. We have already seen that $E$ has the projection property, so we have the desired example.

(b) Archimedean Riesz space with the $Z(\cdot)$-extension property but which is not Dedekind complete

With the notation of example (a), let $E=L_{C}^{b}(I) . Z(E)$ may be identified with $L_{C}^{b}(I)$ in the usual manner. If $H$ is any sublattice of $E$ and $T \in Z(H)$ then there is $\varphi$, defined on $Y=\{x \in I$ : there exists $h \in H$ with $h(x) \neq 0\}$, with

$$
T h(y)=\varphi(y) h(y) \quad(y \in Y) .
$$

Clearly $\varphi$ is bounded and coincides, on some neighbourhood of any given point of $Y$, with a constant function. It may be extended to $\bar{\varphi} \in L_{C}^{b}(I)$ by defining, for example, $D(\bar{\varphi})=S \cup(I \backslash \bar{S})$ and $\bar{\varphi} \mid(I \backslash \bar{S}) \equiv 0$. $\bar{\varphi}$ now defines the required extension, $T$, of $T$. Thus $E$ has the $Z(\cdot)$-extension property, while it is easy to check that it is not Dedekind complete.

We now turn our attention to the space $S(\cdot)$. The relevant condition here is rather different in character from those in all our other results. Note in particular that uniform completeness plays no role here.

THEOREM 2. The following condition on an Archimedean Riesz space $E$ are equivalent:

(i) Every principal ideal in $E$ is finite dimensional.

(ii) There is $a$ set $S$ and a linear order isomorphism, $x \mapsto x^{\wedge}$, of $E$ onto the space of all real-valued functions on $S$ which vanish except on a finite set.

(iii) If $H$ is a sublattice of $E$ and $T \in S(H)$ then there is $T \in S(E)$ with $T \mid H=T$.

Proof. (i) $\Leftrightarrow$ (ii) is Theorem 61.4 of Luxemburg and Zaanen (1971).

(ii) $\Rightarrow$ (iii). Let $R=\left\{s \in S\right.$ : there exists $h \in H$ with $\left.h^{\wedge}(s) \neq 0\right\}$. Each $T \in S(H)$ defines $\varphi \in C^{\infty}(R)(=C(R))$ such that, for $h \in H$,

$$
(T h)^{\wedge}(r)=\varphi(r) h^{\wedge}(r) \quad(r \in R) .
$$

Extend $\varphi$ to $\bar{\varphi}$ on $S$ in any manner. $\bar{\varphi}$ defines $T$ on $E$ by

$$
(T x)^{\wedge}(s)=\bar{\varphi}(s) x^{\wedge}(s) \quad(s \in S)
$$

for each $x \in E$. This is the desired extension of $T$.

(iii) $\Rightarrow$ (i). Let $e \in E^{+}$. If the ideal it generates is not finite dimensional we can find $\left(e_{n}\right)_{n \in \mathbf{N}}$ with $0 \leqslant e_{n} \leqslant e$ and $0 \neq e_{n}$ for all $n \in N$ and $e_{n} \perp e_{m}$ if $n \neq m$. Let $H$ be the linear span of the $e_{n}$ 's, a sublattice of $E$. Define $T$ on $H$ by setting $T e_{n}=n e_{n}$ and extending it linearly. Clearly $T \in S(H)$. Suppose $T$ extends to $T \in S(E)$. We may, by replacing $T$ by its positive part if necessary, assume that $T$ is positive. 
There exists $\lambda>0$ with $T e \leqslant \lambda e$. It follows that $\bar{T} x \leqslant \lambda x$ for all $x$ in the positive part of the ideal generated by $e$. In particular we have $n e_{n}=T e_{n}=T e_{n} \leqslant \lambda e_{n}$, so that $\lambda \geqslant n$ for all $n \in \mathbf{N}$, a contradiction.

This proof shows that $P(E)=S(E)$ for such spaces (it is also true that $P(E)=\operatorname{Stab}(E)$ for them). It is easy to check that the spaces satisfying the equivalent conditions of Theorem 2 are precisely the spaces of all real-valued functions, on some fixed set, which are zero except on a finite set. These spaces have several characterizations, see Section 61 of Luxemburg and Zaanen (1971).

Recall that an Archimedean Riesz space $E$ is universally complete if every disjoint family of positive elements of $E$ has a supremum. The Dedekind complete and universally complete Riesz spaces are precisely those linearly order isomorphic to $C^{\infty}(S)$ for some compact extremally disconnected space $S$. We shall define $E$ to be universally bounded if every disjoint family of positive elements of $E$ has an upper bound. Clearly if $E$ is universally bounded and Dedekind complete it will be universally complete. Example (d) below will be universally bounded and have the projection property but will fail to be universally complete.

THEOREM 3. Consider the following three conditions on an Archimedean Riesz space $E$.

(i) $E$ is Dedekind complete and every principal band is universally complete.

(ii) If $H$ is a sublattice of $E$ and $T \in P(H)$ then there is $T \in P(E)$ with $T \mid H=T$.

(iii) $E$ has the projection property and every principal band is universally bounded.

In general (i) $\Rightarrow$ (ii) $\Rightarrow$ (iii). If $E$ is uniformly complete then all three conditions are equivalent.

Proof. (i) $\Rightarrow$ (ii). Let $x \mapsto x^{\wedge}$ be an admissible representation of $E$ as an ideal in $\mathrm{C}^{\infty}(S)$, where $S$ is a disjoint union of extremally disconnected compact Hausdorff spaces. Let $R=\left\{s \in S\right.$ : there exists $h \in H$ with $\left.0<h^{\wedge}(s)<\infty\right\}$ and let $\varphi \in C^{\infty}(R)$ with, for $h \in H$,

$$
(T h)^{\wedge}(r)=\varphi(r) h^{\wedge}(r)
$$

for all $r \in R$ for which the product is defined. Extend $\varphi$ to $\bar{\varphi} \in C^{\infty}(S)$ (as in Theorem 1). We need only prove that if $x \in E$ then $\bar{\varphi} x^{\wedge} \in E^{\wedge}$ to be able to define $\bar{T} x=y$, where $y^{\wedge}=\bar{\phi} x^{\wedge}$, and deduce that $\bar{T}$ is the desired extension.

Without loss of generality we may assume $\bar{\varphi}$ and $x$ are positive. Define, inductively, open and closed sets $A_{n}$ by

$$
\begin{aligned}
& A_{1}=\{s \in S: \bar{\varphi}(s)<1\}^{-}, \\
& A_{n}=\{s \in S: \bar{\varphi}(s)<n\}^{-} \backslash A_{n-1},
\end{aligned}
$$

so that $n-1 \leqslant \bar{\varphi} \mid A_{n} \leqslant n$. As $E^{\wedge}$ is an ideal in $C^{\infty}(S)$ we have $\bar{\varphi} \chi\left[A_{n}\right] x^{\wedge} \in E^{\wedge}$. Let $z_{n} \in E$ with $z_{n}{ }^{\wedge}=\bar{\varphi} \chi\left[A_{n}\right] x^{\wedge}$. Define $z$ to be the supremum of the $z_{n}$ 's, 
which exists as each $z_{n}$ lies in the band generated by $x$, which band is universally complete. Note that

$$
z^{\wedge}\left|A_{n} \geqslant z_{n}^{\wedge}\right| A_{n}=\bar{\varphi} x^{\wedge} \mid A_{n}
$$

so that $z^{\wedge} \geqslant \bar{\varphi} x^{\wedge}$ by continuity. That $\bar{\varphi} x^{\wedge} \in E^{\wedge}$ now follows as $\bar{\varphi} x^{\wedge} \in C^{\infty}(S)$ and $E^{\wedge}$ is an ideal in $C^{\infty}(S)$ (in fact $\bar{\varphi} x=z^{\wedge}$ ).

(ii) $\Rightarrow$ (iii). The implication (iii) $\Rightarrow$ (iv) of Theorem 1 shows that $E$ has the projection property.

Let $e \in E^{+}$and $\left(x_{\gamma}\right)$ be a disjoint family of positive elements in the band generated by $e$. If $n \in \mathbf{N}$ let $P_{n, \gamma}$ be the band projection onto the band generated by $x_{\gamma} \wedge n e$ and $Q_{n, \gamma}=P_{n, \gamma}-P_{(n-1), \gamma}$ which is also a band projection. Note that $Q_{n, \gamma} x_{\gamma} \leqslant n e$ and that $x_{\gamma}=\sup _{n} Q_{n, \gamma} x_{\gamma}$. The family of all $Q_{n, \gamma} x_{\gamma}$ is disjoint so its linear span is a sublattice $H$ of $E$. Define $T \in P(H)$ with $T\left[Q_{n, \gamma} x_{\gamma}\right]=n\left[Q_{n, \gamma} x_{\gamma}\right]$. Let $T$ be any positive extension of $T$ to the whole of $E$ (if $T$ has an extension in $P(E)$ take its positive part). Now

$$
n Q_{n, \gamma} x_{\gamma}=T\left[Q_{n, \gamma} x_{\gamma}\right] \leqslant n T e
$$

so that $Q_{n, \gamma} x_{\gamma} \leqslant \bar{T} e$ and hence $x_{\gamma} \leqslant \bar{T} e$ for all $\gamma$.

Finally it is clear that (iii), together with uniform completeness, implies (i).

Neither implication (ii) $\Rightarrow$ (i) nor (iii) $\Rightarrow$ (ii) is valid without the assumption of uniform completeness. We shall refer to (ii) as the $P(\cdot)$-extension property.

(c) Archimedean Riesz space with the projection property and which is universally complete but does not have the $P(\cdot)$-extension property

In example (a) replace $E$ by $L_{F}(I)$ and $H$ by $L_{G}(I)$. The only extra comment needed is that every space $L_{A}(I)$ is universally complete.

(d) Archimedean Riesz space with the $P(\cdot)$-extension property which has a weak order unit but is not universally complete (and hence not Dedekind complete)

Let $P$ be the space of piecewise affine functions on $I=[0,1]$. That is those $f \in C(I)$ such that there exist $x_{0}=0, x_{1}, \ldots, x_{n-1}, x_{n}=1$ with $f \mid\left[x_{i}, x_{i+1}\right]$ affine $(0 \leqslant i \leqslant n)$. Let $E$ be the sublattice of $L_{P}(I)$ consisting of those $f$ which coincide with a product $g h\left(g \in L_{C}(I), h \in P\right)$ where it is defined (so $E$ contains the functions with only finitely many slopes).

It is not difficult to check that $P(E)$ consists of multiplication by functions in $L_{C}(I)$. If $H$ is any sublattice of $E$ and $T \in P(H)$ it is represented by a function $\varphi$ on $Y=\left\{x \in I\right.$ : there exists $h \in H$ with $\left.0 \neq h^{\wedge}(x)\right\}$, which it is not difficult to check lies in $L_{C \mid Y}(Y)$. Defining $D(\bar{\varphi})=D(\varphi) \cup(I \backslash \bar{S})$ and $\bar{\varphi} \mid I \backslash \bar{S} \equiv 0$ gives us an extension of $\varphi$ in $L_{C}(I)$, which defines the extension $T$ of $T$ that is required. 
Define $f_{n} \in E$ by $D\left(f_{n}\right)=I \backslash\{1 / n, 1 /(n-1)\}$ and

$$
f_{n}(t)= \begin{cases}n t-1 & (t \in(1 / n, 1 /(n-1)), \\ 0 & (t \in[0,1 / n) \cup(1 /(n-1), 1]) .\end{cases}
$$

It may easily be checked that $\left(f_{n}\right)_{n \in \mathbf{N}}$ has no supremum in $E$.

We come now to our final theorem. The proof differs from our previous ones as we do not have a representation of general orthomorphisms available to us. Note that we do not need uniform completeness for this theorem, but we do need the additional assumption of order density of the sublattice $H$.

TheOREM 4. Let E be an Archimedean Riesz space with the principal projection property and with all principal bands universally complete. If $H$ is an order dense sublattice of $E$ and $T \in S t a b(H)$ then there is $T \in S t a b(E)$ with $\bar{T} \mid H=T$.

Proof. Let $\mathscr{P}=\{(F, S): F$ is a linear subspace of $E, H \subset F, S: F \rightarrow E$ is linear, $\left.S\right|_{H}=T$ and $x \in F, y \in E$ with $x \perp y$ implies $\left.S x \perp y\right\} . P \neq \varnothing$ as $(H, T) \in P$, for if $y \in E^{+}$ then $y$ is the supremum (in $E$ ) of $\left\{y^{\prime} \in H: 0 \leqslant y^{\prime} \leqslant y\right\}$ (using the order density of $H$ in $E$ ). Thus if $x \in H$ and $x \perp y$ we have $x \perp y^{\prime}$ whenever $0 \leqslant y^{\prime} \leqslant y, y^{\prime} \in H$. Hence $T x \perp y^{\prime}$, for all such $y^{\prime}$, and hence $T x \perp y$.

Order $\mathscr{P}$ by defining $(F, S)<\left(F^{\prime}, S^{\prime}\right)$ to mean $F \subset F^{\prime}$ and $S=S^{\prime} \mid F$. If $\left(F_{\gamma}, S_{\gamma}\right)_{\gamma \in \Gamma}$ is a chain in $\mathscr{P}$ let $G=\bigcup_{\gamma \in \Gamma} F$ and define $R$ on $G$ by $R(g)=S_{\gamma}(g)$ if $g \in F_{\gamma}$. This is a well-defined linear mapping. If $g \in G$ and $y \in E$ with $g \perp y$ then $g \in F_{\gamma}$ so that $y \perp S_{\gamma} g=R g$. Thus $(G, R) \in \mathscr{P}$ and it is clear that $(G, R)$ is the supremum in $\mathscr{P}$ of the given chain. It follows from Zorn's lemma that there is a maximal element $(E, T)$ of $\mathscr{P}$.

We claim $E=E$, from which it will follow that $\bar{T}$ is the desired extension. Note first that we may, for each $g \in E$, assume that $P_{g} E \subset E$, where $P_{g}$ denotes the band projection onto the band generated by $g$. For define $S$ on $P_{g} \bar{E} \oplus\left(I-P_{g}\right) \bar{E}$ by

$$
S\left[P_{g} f+\left(f^{\prime}-P_{g} f^{\prime}\right)\right]=P_{g}(\bar{T} f)+\left(\bar{T} f^{\prime}-P_{g}\left(\bar{T} f^{\prime}\right)\right) .
$$

$S$ is well defined, for

implies

$$
P_{g} f+\left(f^{\prime}-P_{g} f^{\prime}\right)=P_{g} h+\left(h^{\prime}-P_{g} h^{\prime}\right)
$$

$$
P_{g}(f-h)=0=\left(I-P_{g}\right)\left(f^{\prime}-h^{\prime}\right) .
$$

Thus $f-h \perp g$ so $T(f-h) \perp g$ and $P_{g}(T f)=P_{g}(T h)$, and $f-h$ lying in the band generated by $g$ implies that $T(f-h)$ is there also, so that $\left(I-P_{g}\right)\left(\bar{T} f^{\prime}\right)=\left(I-P_{g}\right)\left(\bar{T} h^{\prime}\right)$. Clearly also $S$ has the required orthogonality property, so that $S=\bar{T}$ by maximality.

Let $g \in E \backslash E$, contrary to our claim. Without loss of generality we may suppose the band generated by $g$ is the whole of $E$. For if we can extend $\bar{T} \mid P_{g} \bar{E}$ to $P_{g} \bar{E} \oplus \mathbf{R g}$ in the appropriate manner, the extension to $\bar{E} \oplus \mathbf{R g}$ is routine. 
If $y \in E, f \in E$ and $f+g \perp y$, define

$$
P(y, f, g)=-P_{y}(T f),
$$

where $P_{y}$ denotes the band projection onto the band generated by $y$. Note that if also $y^{\prime} \in E, f^{\prime} \in E$ with $f^{\prime}+g \perp y^{\prime}$ then

$$
P_{y} P\left(y^{\prime}, f^{\prime}, g\right)=P_{y^{\prime}} P(y, f, g) .
$$

For $f+g, f^{\prime}+g \perp|y| \wedge\left|y^{\prime}\right|$ imply that $f-f^{\prime} \perp|y| \wedge\left|y^{\prime}\right|$, so that $T\left(f-f^{\prime}\right) \perp|y| \wedge\left|y^{\prime}\right|$ and hence $P_{|y| \wedge\left|y^{\prime}\right|}(\bar{T} f)=P_{|y| \wedge\left|y^{\prime}\right|}\left(T f^{\prime}\right)$. As we also have $P_{y} P_{y^{\prime}}=P_{|y| \wedge\left|y^{\prime}\right|}=P_{y^{\prime}} P_{y}$ we see that

$$
\begin{aligned}
P_{y} P\left(y^{\prime}, f^{\prime}, g\right) & =-P_{y} P_{y^{\prime}}\left(T f^{\prime}\right) \\
& =-P_{|y| \wedge\left|y^{\prime}\right|}\left(T f^{\prime}\right) \\
& =-P_{|y| \wedge\left|y^{\prime}\right|}(T f) \\
& =-P_{y^{\prime}} P_{y}(T f) \\
& =P_{y^{\prime}} P(y, f, g) .
\end{aligned}
$$

I now claim that there is $h \in E$ with

$$
P_{y} h=P(y, f, g)
$$

whenever $y \perp f+g(y \in E, f \in E)$. Let $\left(y_{\gamma}\right)_{\gamma \in \Gamma}$ be a maximal disjoint family with $y_{\gamma} \perp f_{\gamma}+g$ for some $f_{\gamma} \in \bar{E}$. Certainly the family $\left(P\left(y_{\gamma}, f_{\gamma}, g\right)\right)_{\gamma \in \Gamma}$ is disjoint. Using the universal completeness of the band generated by $g(=E)$ we may form

$$
h=\underset{\gamma \in \Gamma}{\bigvee} P\left(y_{\gamma}, f_{\gamma}, g\right)^{+}-\underset{\gamma \in \Gamma}{\bigvee} P\left(y_{\gamma}, f_{\gamma}, g\right)^{-} .
$$

Clearly $P_{\nu_{\gamma}} h=P\left(y_{\gamma}, f_{\gamma}, g\right)$ for each $\gamma \in \Gamma$. Suppose $y \perp f+g$, with $y \in E, f \in E$, then we have seen that

$$
\begin{aligned}
P_{y_{\gamma}} P(y, f, g) & =P_{y} P\left(y_{\gamma}, f_{\gamma}, g\right) \\
& =P_{y} P_{y_{\gamma}} h \\
& =P_{y_{\gamma}} P_{y} h .
\end{aligned}
$$

If there is $z \in E$ with $|z| \leqslant|y|$ but $z \perp y_{\gamma}$ for each $\gamma \in \Gamma$, then $z \perp f+g$ contradicting the maximality of the disjoint family $\left(y_{\gamma}\right)_{\gamma \in \Gamma}$. It follows that, as both $P_{y} h$ and $P(y, f, g)$ lie in the band generated by all the $y_{\gamma}$ 's, $P_{y} h=P(y, f, g)$ as claimed.

Now define $E_{0}=E \oplus \mathbf{R}_{g}, T_{0}(f+\alpha g)=T f+\alpha h$. If $f+\alpha g \perp y \in E$ then either $\alpha=0$, 
so that $f \perp y \Rightarrow T f \perp y \Rightarrow T_{0}(f+0 \cdot g) \perp y$, or else $\alpha^{-1} f+g \perp y \Rightarrow$

$$
\begin{aligned}
P_{y} T_{0}\left(\alpha^{-1} f+g\right) & =P_{y}\left(\bar{T}\left(\alpha^{-1} f\right)+h\right) \\
& =P_{y}\left(\bar{T}\left(\alpha^{-1} f\right)\right)+P_{y} h \\
& =P_{y}\left(\bar{T}\left(\alpha^{-1} f\right)\right)+P\left(y, \alpha^{-1} f, g\right) \\
& =0,
\end{aligned}
$$

so that $T_{0}(f+\alpha g)=\alpha T_{0}\left(\alpha^{-1} f+g\right) \perp y$ again. This contradicts the maximality of $\bar{T}$, completing the proof.

(e) Example to show that order density cannot be dropped from the hypotheses of Theorem 4

The example given by Meyer of an Archimedean Riesz space $E$ with $\operatorname{Stab}(E) \neq P(E)$ is a lattice of real-valued functions on $[0,1]$. This is a sublattice of $\mathscr{F}([0,1])$, the lattice of all real-valued functions on $[0,1]$, which is Dedekind complete and universally complete. Suppose each $T \in S t a b(E)$ extended to $T \in \operatorname{Stab}(\mathscr{F}([0,1]))$. As $\operatorname{Stab}(\mathscr{F}([0,1]))=P(\mathscr{F}([0,1]))$ (if $f(t)=0$ then $f \perp \chi[\{t\}]$ implies $T f \perp \chi[\{t\}]$, which observation makes the representation of elements of $\operatorname{Stab}(\mathscr{F}([0,1]))$ as multiplication by functions on $[0,1]$ simple) we can form $T^{+} \in P(\mathscr{F}([0,1]))$. But if $x \in E^{+}$then $\bar{T}^{+} x=(\bar{T} x)^{+}=(T x)^{+} \in E$. Thus $\bar{T}^{+} \mid E=T^{+}$ exists in $P(E)$, and hence $T \in P(E)$. This contradicts $\operatorname{Stab}(E) \neq P(E)$.

(f) Example of an order bounded orthomorphism with an extension but no order bounded one

Let us return to the example $E=L_{F}(I), H=L_{G}(I)$ and take any $T \in P(H)$ which does not extend to an element of $P(E) . L_{F}(I)$ has the projection property and is universally complete, so we may certainly apply Theorem 4 to extend $T$ to $T \in \operatorname{Stab}(E)$.

(g) Example of a Dedekind complete, universally complete Riesz space $M$ with $P(M) \neq \operatorname{Stab}(M)$

Every Archimedean Riesz space $L$ can be embedded as an order dense sublattice of a Dedekind complete, universally complete Riesz space $M$ (Luxemburg and Zaanen (1971), Theorem 50.8). This applies in particular to Meyer's space or to $L_{F}(I)$, both of which have a non order bounded orthomorphism on them. If we extend this by Theorem 4 the argument used in (e) shows that this extension cannot be order bounded. 


\section{Acknowledgement}

I should like to thank M. Meyer for a number of helpful suggestions concerning this paper, especially the present short proof of implication (iii) $\Rightarrow$ (iv) in Theorem 1 , and also the referee who suggested, in particular, the present proof of (i) $\Rightarrow$ (ii) in Theorem 1 .

\section{References}

A. Bigard and K. Keimel (1969), 'Sur les endomorphismes conservant les polaires d'un groupe réticulé Archimédien', Bull. Soc. Math. France 97, 381-398.

P. F. Conrad and J. E. Diem (1971), 'The ring of polar preserving endomorphisms of an abelian lattice ordered group', Illinois J. Math. 15, 224-240.

W. A. J. Luxemburg (1977), private communication.

W. A. J. Luxemburg and A. C. Zaanen (1971), Riesz spaces I (North-Holland, Amsterdam, London).

M. Meyer (1977), 'Quelques propriétés des homomorphismes d'espaces vectoriels réticulés', preprint.

A. W. Wickstead (1977), 'Representation and duality of multiplication operator on Archimedean Riesz spaces', Compositio Math. 35, 225-238.

A. C. Zaanen (1975), 'Examples of orthomorphisms', J. Approximation Theory 13, 192-204.

Department of Pure Mathematics

The Queen's University of Belfast

Belfast BT7 1NN

Northern Ireland 